\title{
Verification of Higher-Order Computation: A Game-Semantic Approach
}

\author{
C.-H.L. Ong \\ Oxford University Computing Laboratory \\ users.comlab.ox.ac.uk/luke.ong/
}

\begin{abstract}
We survey recent developments in an approach to the verification of higher-order computation based on game semantics. Higherorder recursion schemes are in essence (programs of) the simply-typed lambda calculus with recursion, generated from uninterpreted first-order symbols. They are a highly expressive definitional device for infinite structures such as word languages and infinite ranked trees. As applications of a representation theory of innocent strategies based on traversals, we present a recent advance in the model checking of trees generated by recursion schemes, and the first machine characterization of recursion schemes (by a new variant class of higher-order pushdown automata called collapsible pushdown automata). We conclude with some speculative remarks about reachability checking of functional programs. A theme of the work is the fruitful interplay of ideas between the neighbouring fields of semantics and verification.
\end{abstract}

Game semantics has emerged as a powerful paradigm for giving semantics to a variety of programming languages and logical systems. It has been used to construct the first syntax-independent fully abstract models for a spectrum of programming languages ranging from purely functional languages to languages with non-functional features such as control operators and locally-scoped references 32645251230 etc. In this extended abstract, we present in brief recent developments in algorithmic game semantics, which is concerned with applying game semantics to computer-assisted verification and program analysis 22193633 34.

Game semantics has several features which make it very promising for such applications. It provides a very concrete way of building fully abstract models. It has a clear operational content, which admits compositional methods in the style of denotational semantics. The basic objects studied in game semantics are games (between two players, called $P$ and $O$ ), and strategies on games. As strategies can be seen as certain kinds of highly-constrained processes, they admit the same kind of automata-theoretic representations central to model checking and allied methods in computer-assisted verification [4314]. Moreover games and strategies naturally form themselves into intricate mathematical structures that give very accurate models of advanced high-level programming languages, as the various full abstraction results show. For an introduction to game semantics, see for example [6]. 


\section{Traversal: A Representation Theory of Innocent Strategies}

In game semantics, programs are modelled as $P$-strategies. Strategies, which are certain sets of plays (or legal positions), are typically composed by parallel composition plus hiding, in the sense of the process algebra CSP [24]. The starting point of our work is a representation theory of the game semantics of higher-type programs (such as recursion schemes, PCF and Idealized Algol) that is very concrete, involving combinatorics over infinite structures defined by the abstract syntax trees of the programs being modelled. Take a program $M$ which may be open. In this approach the strategy-denotation of $M$, written $\llbracket M \rrbracket$, is represented by a set $\mathcal{T} r(M)$ of traversals over a possibly infinite tree - called the computation tree of $M$ - which is generated from (a souped up version of) the abstract syntax tree of $M$. (Formally a traversal over a tree is a sequence of nodes starting from the root; quite unlike a path in the tree, a traversal can "jump" all over the tree, and may visit certain nodes infinitely often.) A traversal over the computation tree of $M$ does not correspond to a play in $\llbracket M \rrbracket$, but rather to an interaction sequence that is obtained by uncovering [26] a play in « $M \rrbracket$ in a hereditary fashion; and a suitable projection of $\mathcal{T} r(M)$ - corresponding to the operation of hiding - gives the strategy-denotation $\llbracket M \rrbracket$. We call such a result a Path-Traversal Correspondence Theorem. (Denoting programs by sets of interaction sequences obtained by hereditary uncovering was first considered by Greenland in his DPhil thesis [20, which he has called revealed semantics.) The set $\operatorname{Tr}(M)$ is defined by recursion over the syntax of $M$ and by rule induction. Intuitively these formation rules define what amounts to the composition algorithm of innocent strategies (less the hiding) but expressed in a setting in which moves (of the innocent game) are mapped to nodes of the computation tree. In [12] (see also Blum's forthcoming DPhil thesis [10]) we give a self-contained account of the traversal-based representation theory and establish Path-Traversal Correspondence Theorems for a number of higher-order languages including recursion schemes and PCF.

In the following we consider (higher-order) recursion schemes as a definitional device for infinite structures (mainly ranked trees, but also word languages and directed graphs). We sketch two applications of a Path-Correspondence Theorem for recursion schemes: the first concerns the verification of (possibly infinite) ranked trees generated by recursion schemes, and the second is a machine characterization of recursion schemes.

Recursion schemes of order 1, originally known as recursive program schemes, were first formalized and studied in the early 70's [1735] (although the basic ideas of program schemes and fixpoint theory go further back to David Park in the late 60's); they were an influential formalism for the semantical analysis of both imperative and functional programs 3515. We fix a (ranked) alphabet $\Sigma$. Types are generated from a base type $o$ using the arrow constructor $\rightarrow$. A (higherorder) recursion scheme is a finite set of equations of the form $F x_{1} \cdots x_{n}=e$, where $F: A_{1} \rightarrow \cdots \rightarrow A_{n} \rightarrow o$ is a typed non-terminal, each $x_{i}: A_{i}$ is a typed variable, and $e$ is an applicative term of type $o$ constructed from the 
non-terminals (which include a distinguished start symbol), terminals (which are the $\Sigma$-symbols), and variables $x_{1}, \cdots, x_{n}$. The scheme is said to be order- $k$ if the highest order of the non-terminals is $k$. We use (deterministic) recursion schemes here as generators of possibly infinite term-trees. The tree generated by a recursion scheme is defined to be the (possibly infinite) term-tree built up from the first-order terminal symbols by applying the (equations qua) rewrite rules ad infinitum, replacing the formal parameters by the actual parameters, starting from the start symbol. Note that in essence, recursion schemes are programs of the simply-typed lambda calculus with recursion (generated from uninterpreted 1st-order symbols).

\section{Model-Checking Trees Generated by Recursion Schemes}

In a FOSSACS'02 paper [28], Knapik, Niwiński and Urzyczyn studied the infinite hierarchy of term-trees generated by higher-order recursion schemes that are homogeneously typed and satisfy a syntactic constraint called safety 1 . They showed that for every $n \geq 0$, the trees that are generated by order- $n$ safe schemes have decidable monadic second-order (MSO) theories. Later in the year at MFCS'02 13. Caucal introduced a tree hierarchy and a graph hierarchy that are defined by mutual recursion, using a pair of powerful transformations that preserve decidability of MSO theories. Caucal's tree hierarchy coincides with the hierarchy of trees generated by higher-order safe recursion schemes. In 28] Knapik et al. asked if the safety assumption is really necessary for their MSO decidability result. A partial answer was subsequently obtained by Aehlig, de Miranda and Ong; in a TLCA'05 paper [7, they showed that trees that are generated by order-2 recursion schemes, whether safe or not, have decidable MSO theories. Independently, Knapik, Niwiński, Urzyczyn and Walukiewicz obtained a sharper result: in an ICALP'05 paper 29, they proved that the modal mu-calculus model-checking problem for trees generated by order-2 recursion schemes (whether safe or not) is 2-EXPTIME complete. A year later in a LICS'06 paper [37, we gave a complete answer to the question:

Theorem 1 (Decidability). The modal mu-calculus model-checking problem for trees generated by order-n recursion schemes (whether safe or not, and whether homogeneously typed or not) is n-EXPTIME complete, for every $n \geq 0$. Thus these trees have decidable MSO theories.

Our approach to the decidability result is to transfer the algorithmic analysis from the tree generated by a recursion scheme, which we call value tree, to the computation tree, which is itself a tree generated by a related order-0 recursion scheme (equivalently, a regular tree). The computation tree recovers useful intensional information about the computational process behind the construction of the value tree. Paths in the value tree correspond exactly to plays in the game

\footnotetext{
${ }^{1}$ The safety condition may be presented as a set of rules that determine where a variable may occur as a subterm of a term, depending on both the order of the variable and the order of the term (see [1110]).
} 
semantics of the recursion scheme; a traversal is then (a representation of) the uncovering of such a play. By appealing to the Path-Traversal Correspondence Theorem, we prove that a given alternating parity tree automaton (APT) [18] has an accepting run-tree over the value tree if and only if it has an accepting traversal-tree over the computation tree. Our problem is then reduced to finding an effective way of recognizing a set of infinite traversals (over a given computation tree) that satisfy the parity condition. This requires a new idea as a traversal is most unlike a path. Our solution again exploits the game-semantic connection. It is a property of traversals that their P-views are paths (in the computation tree). This allows us to simulate a traversal over a computation tree by (the P-views of its prefixes, which are) annotated paths of a certain kind in the same tree. The simulation is made precise in the notion of traversal-simulating APT. We establish the correctness of the simulation by proving that a given property APT has an accepting traversal-tree over the computation tree if and only if the associated traversal-simulating APT has an accepting run-tree over the computation tree. Note that the decidability of the modal mu-calculus model-checking problem for trees generated by recursion schemes follows at once since computation trees are regular, and the APT acceptance problem for regular trees is decidable 4018 .

\section{A Machine Characterization of Higher-Order Recursion Schemes}

Another application of the Path-Traversal Correspondence Theorem concerns a fundamental question about higher-order recursion schemes: Can we characterize their expressivity by a class of machine models? Knapik, Niwiński and Urzyczyn [28] have shown that as generators of ranked trees, higher-order safe recursion schemes are equi-expressive with higher-order pushdown automata 31. Their result and an earlier result by Damm and Goerdt [16] may be viewed as attempts to answer the question; they both had to impose somewhat unnatural syntactic constraints (of safety and derived types respectively) on recursion schemes in order to establish their characterizations.

A partial answer was recently obtained by Knapik, Niwiński, Urzyczyn and Walukiewicz. In an ICALP'05 paper 29], they proved that order-2 homogeneouslytyped (but not necessarily safe) recursion schemes are equi-expressive with a variant class of order-2 pushdown automata called panic automata. In a preprint [21, we give a complete answer to the question. We introduce a new kind of higher-order pushdown automata (which generalize pushdown automata with links [8], or equivalently panic automata, to all finite orders), called collapsible pushdown automata (CPDA), in which every symbol in the stack has a link to a (necessarily lowerordered) stack situated somewhere below it. In addition to the higher-order stack operations push $_{i}$ and pop $_{i}$, CPDA have an important operation called collapse, whose effect is to "collapse" a stack $s$ to the prefix as indicated by the link from the top $_{1}$-symbol of $s$. In [21] we prove the following result:

${ }^{2}$ Property APT because the APT corresponds to the property described by a given modal mu-calculus formula. 
Theorem 2 (Equi-Expressivity). CPDA are equi-expressive with recursion schemes as generators of (possibly infinite) ranked trees.

In one direction, we give a simple algorithm that transforms an order- $n$ CPDA to an order- $n$ recursion scheme that generates the same tree, uniformly for all $n \geq 0$. In the other direction, using ideas from game semantics, we give an effective transformation of order- $n$ recursion schemes (not assumed to be homogeneously typed, and hence not necessarily safe) to order- $n$ CPDA that compute traversals over the computation tree of the scheme, and hence paths in the tree generated by the scheme. Our equi-expressivity result is the first automata-theoretic characterization of higher-order recursion schemes. Thus CPDA are also a characterization of the simply-typed lambda calculus with recursion (generated from uninterpreted 1st-order symbols) and of (pure) innocent strategies.

\section{Verifying PCF Programs: Reachability Checking}

As a further direction (and a possible application of path-traversal correspondence), we consider the problem of reachability checking of higher-order computation. In the simplest form, reachability is the problem: Given a state of a transition system, is it reachable from the start state? Reachability is arguably the most important test in the computer-assisted verification of computing systems. Reachability (in its various forms) is expressible in standard temporal logics such as EF, LTL, CTL, etc., but it is typically computationally more tractable than the model checking of any of these logics (e.g. for pushdown systems, reachability is polytime [1, whereas EF-, LTL- and CTL-model checking are respectively PSPACE-complete, EXPTIME-complete and EXPTIME-complete [27]). In recent years, reachability checkers (such as SLAM [9], Blast [23], etc.) for first-order imperative programs have had a major impact in the verification community. Perhaps because of its simplicity and ease of use, reachability is now a standard approach to checking safety properties in the industry. It is therefore somewhat surprising that no reachability checker has been developed for higher-order programming languages such as Ocaml, Haskell and F\#. Indeed, to our knowledge, reachability of higher-order computation does not appear to have been studied in the literature.

The simplest (though already challenging) setting is PCF (generated from finite base types). We propose the following decision problem:

PCF-Reachability: Given a (possibly open) PCF term $M$ and a subterm $N$ of $M$, is there a program context $C[]$ such that the evaluation of $C[M]$ entails the evaluation of $N$ ? (Precisely, is there a program context $C[]$ such that $C[M] \longrightarrow^{*} E[N]$ for some evaluation context $E[]$ ?)

For which fragment of PCF is the problem decidable? If there are positive answers, it would be interesting to consider the "global version" of the problem i.e. is it possible to compute a finite description of the set of contexts $C$ [] for a given pair of $M$ and $N$ ? 
An approach that seems promising is to appeal to the Path-Traversal Theorem for PCF [10], and consider traversals over the computation tree of $M$. The idea is to use appropriate alternating tree automata to "guess" a set of paths in the computation tree simulating traversals that witness yes-instances of the problem (see [37]). If this works out, it would be interesting to present the algorithm in terms that functional programmers can readily understand and appreciate.

Remark 1. (i) It is not clear if there is any connection between reachability (in our sense) and control flow analysis (e.g. 42]) of functional programs. In the past couple of years there have been several interesting developments in the verification and flow analysis of functional language. $\mathrm{Xu}$ and Peyton Jones have studied contract checking in Haskell (see Xu's forthcoming PhD thesis). A recent project of Shivers et al. 32] used abstract interpretation (specifically abstract counting) to build more precise flow analysers by garbage collecting "dead" environment structure in the abstract state space traversed by the functional programs.

(ii) When restricted to finitary (i.e. recursion-free) PCF, the problem is related to the atoms case of the Interpolation Problem, which is decidable [38. (The Interpolation Problem is equivalent to the Higher-Order Matching Problem 4139.)

\section{References}

1. Bouajjani, A., Esparza, J., Maler, O.: Reachability analysis of pushdown automata: Application to model-checking. In: International Conference on Concurrency Theory, pp. 135-150 (1997)

2. Abramsky, S., Honda, K., McCusker, G.: Fully abstract game semantics for general reference. In: Proceedings of IEEE Symposium on Logic in Computer Science, 1998, Computer Society Press (1998)

3. Abramsky, S., Jagadeesan, R., Malacaria, P.: Full abstraction for PCF. Information and Computation 163 (2000)

4. Abramsky, S., McCusker, G.: Linearity, sharing and state: A fully abstract game semantics for Idealized Algol with active expressions. In: O'Hearn, P.W., Tennent, R.D. (eds.) Algol-like languages, Birkhäuser (1997)

5. Abramsky, S., McCusker, G.: Call-by-value games. In: Nielsen, M. (ed.) CSL 1997. LNCS, vol. 1414, Springer, Heidelberg (1998)

6. Abramsky, S., McCusker, G.: Game semantics. In: Schwichtenberg, H., Berger, U. (eds.) Logic and Computation: Proceedings of the 1997 Marktoberdorf Summer School, Springer, Heidelberg (1998)

7. Aehlig, K., de Miranda, J.G., Ong, C.-H.L.: The monadic second order theory of trees given by arbitrary level two recursion schemes is decidable. In: Urzyczyn, P. (ed.) TLCA 2005. LNCS, vol. 3461, pp. 39-54. Springer, Heidelberg (2005)

8. Aehlig, K., de Miranda, J.G., Ong, C.-H.L.: Safety is not a restriction at level 2 for string languages. In: Proceedings of the 8th International Conference on Foundations of Software Science and Computational Structures (FOSSACS 2005). LNCS, vol. 3411, pp. 490-501. Springer, Heidelberg (2005)

9. Ball, T., Rajamani, S.K.: The SLAM Project: Debugging system software via static analysis. In: Proc. POPL, pp. 1-3. ACM Press, New York (2002) 
10. Blum, W.: The Safe Lambda Calculus. PhD thesis, University of Oxford (in preparation, 2008)

11. Blum, W., Ong, C.-H.L.: Safe lambda calculus. In: Della Rocca, S.R. (ed.) TLCA 2007. LNCS, vol. 4583, pp. 39-53. Springer, Heidelberg (2007)

12. Blum, W., Ong, C.-H.L.: Path-correspondence theorems and their applications (preprint, 2008)

13. Caucal, D.: On Infinite Terms Having a Decidable Monadic Theory. In: Diks, K., Rytter, W. (eds.) MFCS 2002. LNCS, vol. 2420, pp. 165-176. Springer, Heidelberg (2002)

14. Clarke, E.M., Grumberg, O., Peled, D.: Model Checking. MIT Press, Cambridge (1999)

15. Damm, W.: The IO- and OI-hierarchy. Theoretical Computer Science 20, 95-207 (1982)

16. Damm, W., Goerdt, A.: An automata-theoretical characterization of the OIhierarchy. Information and Control 71, 1-32 (1986)

17. de Roever, W.-P., de Bakker, J.W.: A calculus for recursive program schemes. In: Nivat, M. (ed.) Proc. IRIA symposium on Automata, Languages and Programming, North-Holland, Amsterdam (1972)

18. Emerson, E.A., Jutla, C.S.: Tree automata, mu-calculus and determinacy. In: Proceedings of FOCS 1991, pp. 368-377 (1991)

19. Ghica, D.R., McCusker, G.: Reasoning about Idealized ALGOL Using Regular Languages. In: Welzl, E., Montanari, U., Rolim, J.D.P. (eds.) ICALP 2000. LNCS, vol. 1853, pp. 103-116. Springer, Heidelberg (2000)

20. Greenland, W.: Game semantics for region analysis. PhD thesis, Oxford University Computing Laboratory (2005)

21. Hague, M., Murawski, A.S., Ong, C.-H.L., Serre, O.: Collapsible pushdown automata and recursion schemes. Technical report, Oxford University Computing Laboratory, p. 59 (preprint, 2007), http://users.comlab.ox.ac.uk/luke.ong/

22. Hankin, C., Malacaria, P.: A new approach to control flow analysis. In: Koskimies, K. (ed.) CC 1998. LNCS, vol. 1383, pp. 95-108. Springer, Heidelberg (1998)

23. Henzinger, T.A., Jhala, R., Majumdar, R., Sutre, G.: Software verification with BLAST. In: Proc. 10th SPIN Workshop (2003)

24. Hoare, C.A.R.: Communicating Sequential Processes. Prentice-Hall, Englewood Cliffs (1985)

25. Honda, K., Yoshida, N.: Game-theoretic analysis of call-by-value computation (extended abstract). In: Degano, P., Gorrieri, R., Marchetti-Spaccamela, A. (eds.) ICALP 1997. LNCS, vol. 1256, Springer, Heidelberg (1997)

26. Hyland, J.M.E., Ong, C.-H.L.: On Full Abstraction for PCF: I. Models, observables and the full abstraction problem, II. Dialogue games and innocent strategies, III. A fully abstract and universal game model. Information and Computation 163, 285-408 (2000)

27. Walukiewicz, I.: Model Checking CTL Properties of Pushdown Systems. In: Kapoor, S., Prasad, S. (eds.) FST TCS 2000. LNCS, vol. 1974, Springer, Heidelberg (2000)

28. Knapik, T., Niwiński, D., Urzyczyn, P.: Higher-Order Pushdown Trees Are Easy. In: Nielsen, M., Engberg, U. (eds.) ETAPS 2002. LNCS, vol. 2303, pp. 205-222. Springer, Heidelberg (2002)

29. Knapik, T., Niwiński, D., Urzyczyn, P., Walukiewicz, I.: Unsafe Grammars and Panic Automata. In: Caires, L., Italiano, G.F., Monteiro, L., Palamidessi, C., Yung, M. (eds.) ICALP 2005. LNCS, vol. 3580, pp. 1450-1461. Springer, Heidelberg (2005) 
30. Laird, J.: A semantic analysis of control. PhD thesis, University of Edinburgh (1998)

31. Maslov, A.N.: Multilevel stack automata. Problems of Information Transmission 12, 38-43 (1976)

32. Might, M., Chambers, B., Shivers, O.: Model Checking Via $\Gamma$ CFA. In: Cook, B., Podelski, A. (eds.) VMCAI 2007. LNCS, vol. 4349, pp. 59-73. Springer, Heidelberg (2007)

33. Murawski, A., Walukiewicz, I.: Third-Order Idealized Algol with Iteration Is Decidable. In: Sassone, V. (ed.) FOSSACS 2005. LNCS, vol. 3441, pp. 202-218. Springer, Heidelberg (2005)

34. Murawski, A.S., Ong, C.-H.L., Walukiewicz, I.: Idealized Algol with ground recursion and DPDA equivalence. In: Caires, L., Italiano, G.F., Monteiro, L., Palamidessi, C., Yung, M. (eds.) ICALP 2005. LNCS, vol. 3580, pp. 917-929. Springer, Heidelberg (2005)

35. Nivat, M.: On the interpretation of recursive polyadic program schemes. Symp. Math. XV, 255-281 (1975)

36. Ong, C.-H.L.: Observational equivalence of third-order Idealized Algol is decidable. In: Proceedings of IEEE Symposium on Logic in Computer Science, Copenhagen, Denmark, July 22-25, 2002, pp. 245-256. Computer Society Press (2002)

37. Ong, C.-H.L.: On model-checking trees generated by higher-order recursion schemes. In: Proceedings 21st Annual IEEE Symposium on Logic in Computer Science, Seattle, pp. 81-90. Computer Society Press (2006), users.comlab.ox.ac.uk/luke.ong/

38. Padovani, V.: Decidability of all minimal models. In: Berardi, S., Coppo, M. (eds.) TYPES 1995. LNCS, vol. 1158, pp. 201-215. Springer, Heidelberg (1996)

39. Padovani, V.: Decidability of fourth-order matching. Math. Struct. in Comp. Science 10, 361-372 (2000)

40. Rabin, M.O.: Decidability of second-order theories and automata on infinite trees. Trans. Amer. Maths. Soc. 141, 1-35 (1969)

41. Schubert, A.: A linear interpolation for the higher-order matching problem. In: Bidoit, M., Dauchet, M. (eds.) CAAP 1997, FASE 1997, and TAPSOFT 1997. LNCS, vol. 1214, pp. 441-452. Springer, Heidelberg (1997)

42. Shivers, O.: Control-flow analysis of higher-order languages. PhD thesis, CarnegieMellon University (1991)

43. Vardi, M., Wolper, P.: An automata-theoretic approach to automatic program verification. In: Proc. IEEE Annual Symposium on Logic in Computer Science, IEEE Computer Society Press (1986) 